(C) Elsevier Sequoia S.A., Lausanne - Printed in the Netherlands

\title{
AN EMPIRICAL EQUATION INCLUDING FRACTURE TOUGHNESS AND DESCRIBING FRICTION OF DENTAL RESTORATIVE MATERIALS
}

\author{
J. C. ROBERTS, J. M. POWERS and R. G. CRAIG
}

School of Dentistry, The University of Michigan, Ann Arbor, Mich. 48109 (U.S.A.)

(Received April 1, 1977)

\section{Summary}

An equation was developed from studies of single-pass wear and fracture toughness of filled and unfilled dental restorative resins and dental amalgams relating the sliding frictional force $F$ to the normal load $N$, fracture toughness, modulus of elasticity, yield strength and slider diameter. This equation was of the same form as that reported for viscoelastic materials $\left(F=K N^{n}\right)$. Friction was caused primarily by ploughing or deformation of the material under the conditions tested.

\section{Introduction}

Viscoelastic polymers have been shown to obey a relationship between the frictional force $F$ and the normal load $N$ as follows:

$$
F=K N^{n}
$$

where $K$ is a function of the elastic properties of the material, the shear strength, the penetration hardness, adsorbed species and geometry $[1-5]$.

During the wear process energy may also be expended by the formation of new surfaces. This energy, which can be described as fracture toughness, may be another important parameter accounting for the friction of materials. A model has been proposed that relates the wear of metallic materials and fracture toughness [6].

The purpose of this investigation was to develop an empirical equation between the frictional force caused by sliding and the normal load that would include the fracture toughness, elastic properties, yield strength and slider geometry for filled and unfilled dental restorative resins and dental amalgams. The results were correlated with data obtained from sliding and rolling tests on other materials $[1,2,7]$. 
A commercial dental acrylic resin (A), an experimental formulation of a diacrylate resin without filler (B), a commercial dental composite (C), a spherical dental amalgam (D), a lathe-cut dental amalgam (E) and an admixed dental amalgam (F) were evaluated for the mode and extent of surface damage in previous tests $[8,9]$. Product names, batch numbers, manufacturers and sample size are listed in Table 1.

For the measurement of frictional force, the resins and amalgams were mixed according to the manufacturers' instructions and condensed into cylindrical holders as described elsewhere [8,9]. All samples were stored at $37^{\circ} \mathrm{C}$ before testing. The resins were tested after $24 \mathrm{~h}$ and the amalgams were tested after one week.

The apparatus used to scratch the surface of a specimen and to measure the tangential load has been described elsewhere $[10,11]$; it consisted of a surface grinder*, a loading jig, a diamond slider, a friction transducer and a sample holder. A diamond hemisphere ( $360 \mu \mathrm{m}$ in diameter) was slid across the surface of the specimens. For the resins, 14 parallel one-traversal scratches that resulted from sliding a normal load of $2-7 \mathrm{~N}$ were made on each specimen in distilled water. For the amalgams, eight parallel scratches that resulted from sliding normal loads of $2,3,4,5,8,10,12$ and $15 \mathrm{~N}$ were made. The diamond slider was attached to the loading jig by a strain gauge transducer that allowed the tangential force to be measured. The sample holder was mounted on the table of the surface grinder moving at a speed of $0.025 \mathrm{~cm} \mathrm{~s}^{-1}$.

For measurement of fracture toughness $R$, modulus of elasticity $E$ and yield strength $\sigma_{y}$, the resins and amalgams were mixed according to the manufacturers' instructions and condensed into a rectangular hole $(20 \mathrm{~mm}$ long, $4 \mathrm{~mm}$ wide and $2.5 \mathrm{~mm}$ deep) in a four-piece rectangular die ( $38 \mathrm{~mm}$ long, $19 \mathrm{~mm}$ wide and $14 \mathrm{~mm}$ high). Prenotching of the specimens for measurement of $R$ was accomplished by means of a spacer inserted into the die $[12,13]$. The resin and amalgam samples were stored at $37^{\circ} \mathrm{C}$ for $24 \mathrm{~h}$ before testing.

The apparatus used to test the specimens has been described elsewhere $[12,13]$. It consisted essentially of a three-point bending test fixture, testing machine** and chart recorder. Loads were applied to the specimens at a crosshead rate of $0.05 \mathrm{~cm} \mathrm{~min}^{-1}$. Load and deflection data were taken from the chart recorder. These data were used for the calculation of $R, E$ and $\sigma_{\mathrm{y}}$ $[12,13]$.

The Buckingham pi theorem [14] asserts that any complete physical relationship can be represented by a set of independent non-dimensional combinations of the measures of the physical quantities concerned. For this experiment, the physical quantities were frictional force $F$, normal load $N$,

*Grand Rapids 250, Gallmeyer and Livingston Co., Grand Rapids, Michigan, U.S.A.

**Model TT-BM, Instron Corporation, Canton, Mass. 02021, U.S.A. 


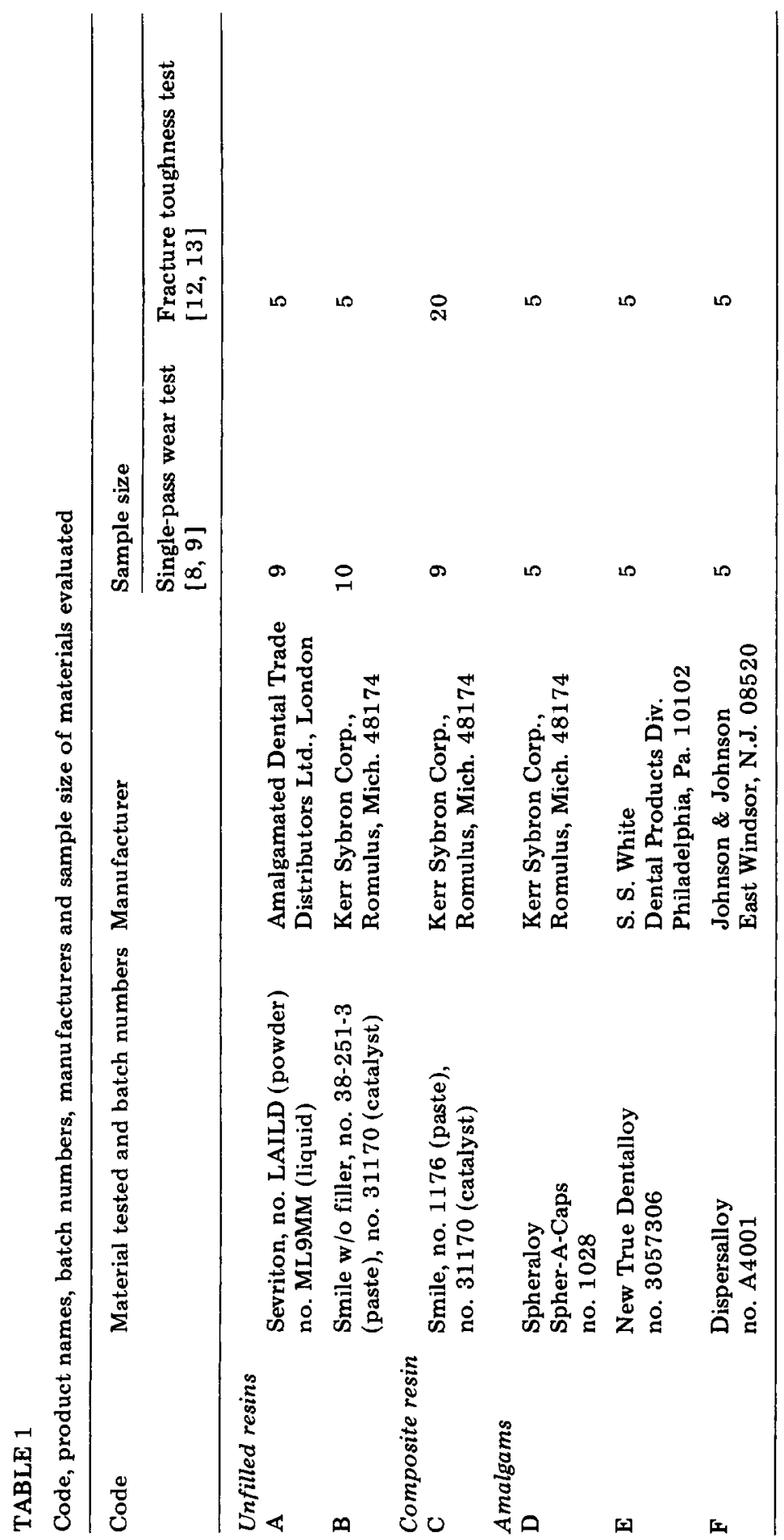




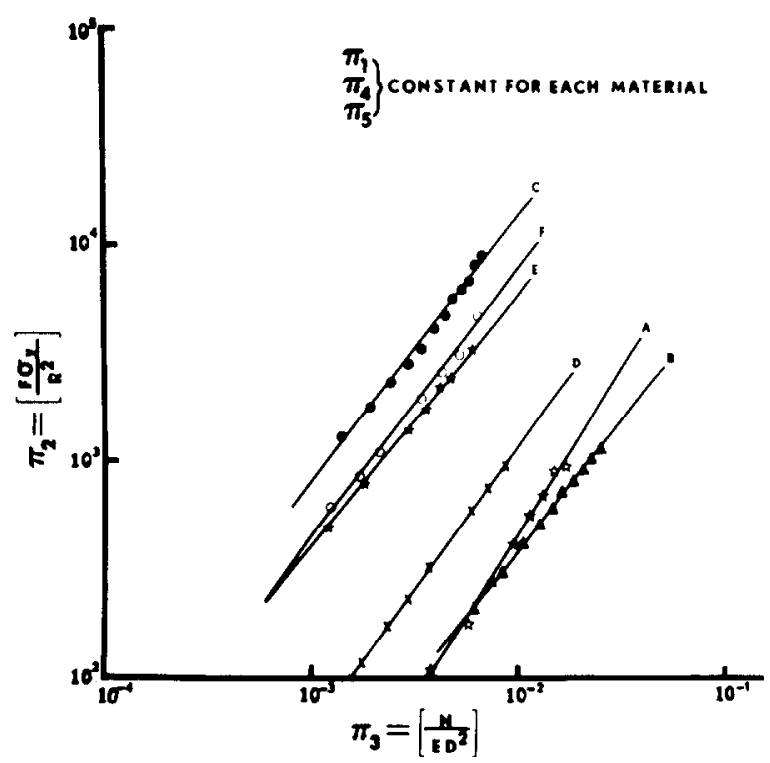

Fig. 1. $\log \pi_{2}$ vs. $\log \pi_{3}$ for materials A - F. All other values of $\pi$ were held constant.

slider diameter $D$, modulus of elasticity $E$, y ield strength $\sigma_{\mathrm{y}}$, fracture toughness $R$ and Poisson's ratio $v$.

The Buckingham pi theorem states that

$$
\pi=f\left(F^{\alpha_{1}}, N^{\alpha_{2}}, D^{\alpha_{3}}, E^{\alpha_{4},} R^{\alpha_{5}}, \sigma_{\mathrm{y}}^{\alpha_{6}}, \nu^{\alpha_{7}}\right)
$$

The force-length-time system of units was used. Since $\nu$ was non-dimensional and constant

$$
\pi_{1}=\nu
$$

Upon substitution of units for the physical quantities involved, the following equations were formed:

$$
\begin{aligned}
& \alpha_{1}+\alpha_{2}+\alpha_{4}+\alpha_{5}+\alpha_{6}=0 \\
& \alpha_{3}-2 \alpha_{4}-2 \alpha_{5}-\alpha_{6}=0
\end{aligned}
$$

Equations (3) and (4) were solved simultaneously by setting one $\alpha_{i}$ to 1 and three others to zero, alternately for different $\alpha_{i}$ s. The following four nondimensional terms were formed:

$$
\begin{aligned}
& \pi_{2}=F \sigma_{\mathrm{y}} / R^{2} \\
& \pi_{3}=N / E D^{2} \\
& \pi_{4}=E / \sigma_{\mathrm{y}} \\
& \pi_{5}=D \sigma_{\mathrm{y}} / R
\end{aligned}
$$

By plotting $\pi_{2}$ versus $\pi_{3}$ with $\pi_{1}, \pi_{4}$ and $\pi_{5}$ constant, a relationship was obtained between the frictional force and the normal load that included the other mechanical variables. 


\section{TABLE 2}

Modulus of elasticity, yield strength, fracture toughness, values of $\pi_{i}, K$ and constants from curve fitting the equation $y=A x^{n}$ to the $\log -\log$ plots of $\pi_{2}$ versus $\pi_{3}$

\begin{tabular}{|c|c|c|c|c|c|c|c|c|c|}
\hline Material & $\begin{array}{l}E^{\mathrm{a}} \\
(\mathrm{GPa})\end{array}$ & $\begin{array}{l}\sigma_{\mathrm{y}}^{\mathrm{a}} \\
(\mathrm{MPa})\end{array}$ & $\begin{array}{l}R^{\mathrm{a}} \\
\left(\mathrm{J} \mathrm{m}^{-2}\right)\end{array}$ & $\pi_{1}^{b}$ & $\pi_{4}$ & $\pi_{5}$ & $A \times 10^{-4}$ & $n$ & $\begin{array}{l}K \\
\left(\mathrm{~N}^{1-n}\right)\end{array}$ \\
\hline A & 2.00 & 104 & 382 & 0.36 & 19.2 & 98.0 & 52.5 & 1.54 & 0.141 \\
\hline B & 1.85 & 65 & 402 & - & 28.5 & 58.2 & 9.23 & 1.19 & 0.338 \\
\hline C & 7.86 & 135 & 182 & 0.28 & 58.2 & 267 & 389 & 1.23 & 0.190 \\
\hline D & 12.9 & 142 & 117 & 0.33 & 90.8 & 437 & 1290 & 1.17 & 0.211 \\
\hline $\mathbf{E}$ & 12.8 & 119 & 247 & 0.33 & 108 & 173 & 471 & 1.31 & 0.146 \\
\hline $\mathbf{F}$ & 17.7 & 130 & 104 & 0.33 & 136 & 450 & 2360 & 1.25 & 0.124 \\
\hline
\end{tabular}

${ }^{\mathrm{a}}$ Taken from Roberts et al. $[12,13]$ at a loading rate of $0.05 \mathrm{~cm} \mathrm{~min}^{-1}$.

${ }^{b}$ Taken from refs. 16, 17.

\section{Results}

$\log -\log$ plots of $\pi_{2}$ versus $\pi_{3}$ with constant values of $\pi_{1}, \pi_{4}$ and $\pi_{5}$ are shown in Fig. 1 for each material. A classical linear regression curve [15] was fitted to the data of the curve of $\log \pi_{2}$ versus $\log \pi_{3}$ for all materials tested. The slope $n$ and the antilogarithm $A$ of the intercept are shown in Table 2.

The information in Fig. 1 indicated that an equation of the form $Y=$ $A x^{n}$ existed between $\pi_{2}$ and $\pi_{3}$. When the physical quantities were substituted for $\pi_{2}$ and $\pi_{3}$, the equation had the form

$$
\frac{F \sigma_{\mathrm{y}}}{R^{2}}=A\left(\frac{N}{E D^{2}}\right)^{n}
$$

When rearranged eqn. (9) became

$$
F=\left(\frac{A R^{2}}{\sigma_{\mathrm{y}} E^{n} D^{2 n}}\right) N^{n}
$$

Equation (10) has the form $F=K N^{n}$, where $K=A R^{2} / \sigma_{\mathrm{y}} E^{n} D^{2 n}$. Values of $E, \sigma_{y}, R, \pi_{1}, \pi_{4}, \pi_{5}, A, n$ and $K$ for materials A - F are listed in Table 2. In this study $D$ was equal to $360 \mu \mathrm{m}$. Plots of frictional force $F$ versus normal load $N$ for materials A - F developed from eqn. (10) are shown in Fig. 2.

\section{Discussion}

The correlation coefficients $r$ for $\log \pi_{2}$ versus $\log \pi_{3}$ for materials A - F were computed [15] to be $0.9984,0.9993,0.9995,0.9996,0.9996$ and 0.9994 , respectively. The critical values of $r$ above which the hypothesis of 


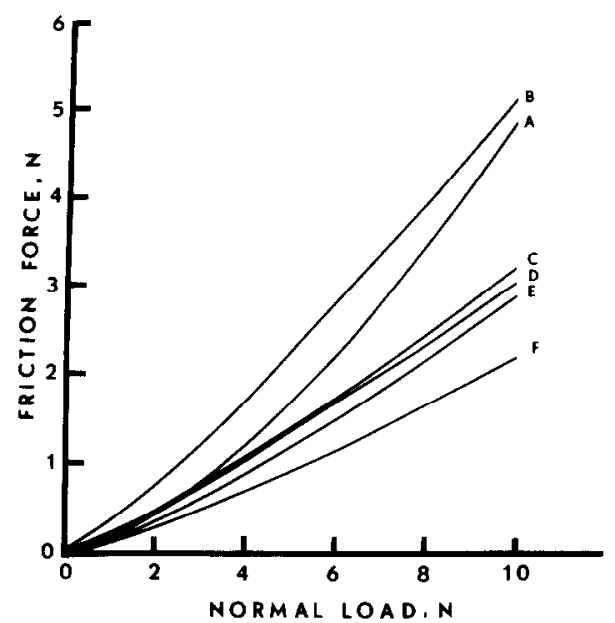

Fig. 2. Sliding frictional force $F$ vs. normal load $N$ for materials A - F.

TABLE 3

Exponents $n$ and $b$ from the equation $F=K^{\prime} N^{n} D^{b}$ for several materials for either rolling or sliding friction ${ }^{\mathrm{a}}$

\begin{tabular}{llll}
\hline Material & $n$ & $b$ & Type of friction \\
\hline Dental restorative materials (A - F) & $1.17-1.54$ & $-2.34-3.08$ & $\begin{array}{l}\text { Sliding with minimal } \\
\text { adhesion component }\end{array}$ \\
Polyethylene [1] & 0.89 & 0.22 & Sliding \\
Nylon [1] & 0.74 & 0.52 & Sliding \\
PTFE [1] & 0.80 & 0.40 & Sliding \\
PTFE [2] & 1.55 & -1.1 & Rolling \\
Wood [7] & $1.33-1.45$ & $-0.75--0.80$ & Rolling \\
Theoretical [2] & 0.89 & 0.22 & Sliding \\
Theoretical [2] & 1.50 & -1.0 & Rolling \\
\hline
\end{tabular}

${ }^{a} F$ is the frictional force, $N$ is the normal load, $D$ is the slider or roller diameter and $K^{\prime}=$ $K / d^{b}$, where $K$ and $b$ are constants.

independence of $\log \pi_{2}$ and $\log \pi_{3}$ could be rejected were $0.7067,0.6319$, $0.5529,0.6664,0.7067$ and 0.7067 for materials A - F, respectively, at the 95\% confidence level. This correlation establishes a relationship between frictional force $F$ and normal load $N$ of the form $F=K N^{n}$. If this equation is rewritten in terms of the slider diameter $D$, it results in $F=K^{\prime} N^{n} D^{b}$, where $K^{\prime}=K / D^{b}$. Table 3 gives values of $n$ and $b$ for the dental restorative materials along with polyethylene [1], nylon [1], polytetrafluoroethylene (PTFE) $[1,2]$ and wood [7] for sliding and rolling friction.

Theoretical equations for conditions of sliding friction $F_{\mathrm{s}}$, where the friction is equal to the force necessary to shear adhesive junctions, and rolling friction $F_{\mathrm{r}}$, where the friction is equal to the force necessary to deform the surface, have been developed by Tabor and Williams [2]. Theoretical 


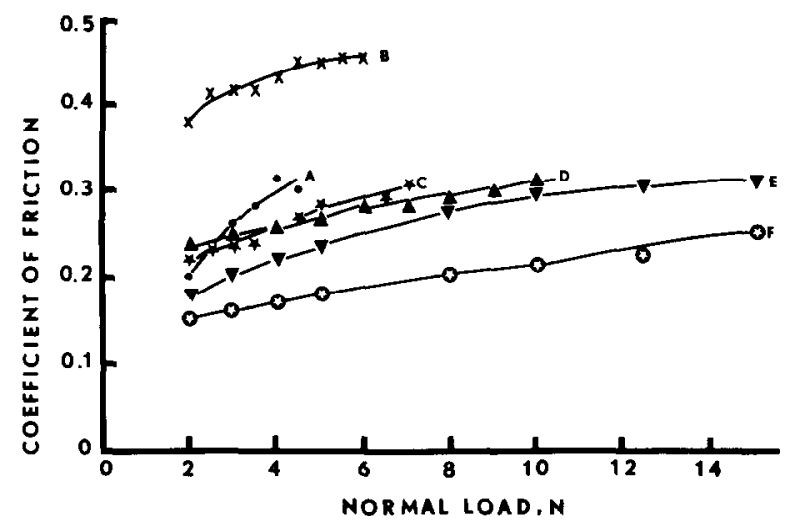

Fig. 3. Coefficient of sliding friction $\mu$ vs. normal load $N$ for materials $\mathrm{A}$ - F.

values of $n$ and $b$ give $0<n<1$ and $b>0$ for sliding friction and $n>1$ and $b<0$ for rolling friction.

The dental restorative materials under conditions of sliding have values of $n$ and $b$ that appear to fit the model of rolling friction. These values can be explained by noting that the frictional force $F_{\mathbf{s}}$ caused by sliding can be separated into two components, the force $F_{\mathrm{d}}$ caused by deformation and the force $F_{\mathrm{a}}$ caused by adhesion, so that $F_{\mathrm{s}}=F_{\mathrm{d}}+F_{\mathrm{a}}$. If the adhesive component is very small, $F_{\mathrm{d}}$ is essentially equal to the force $F_{\mathrm{r}}$ described by rolling. Therefore, it appears that when a hemispherical diamond slider is slid on the dental restorative materials in the presence of distilled water, the adhesive component of the force is small and friction is caused mainly by the deformation or ploughing component of the force.

Plots of the coefficient of friction $\mu$ versus normal load $N$ for materials A - F are shown in Fig. 3. The coefficient of friction increases with increasing normal load over the range of loads studied for these materials. The correlation coefficients $r$ for $\log \mu$ versus $\log N$ for materials $\mathrm{A}$ - F were $0.9878,0.9734,0.9878,0.9823,0.9943$ and 0.9738 , respectively. The critical values of $r$ above which the hypothesis of independence of $\log \mu$ and $\log N$ could be rejected were $0.7067,0.6319,0.5529,0.6664,0.7067$ and 0.7067 for materials $\mathrm{A}-\mathrm{F}$, respectively, at the $95 \%$ confidence level. Therefore, the relationship between $\mu$ and $N$ is of the form $\mu=K N^{c}$, where $K$ and $c$ are constants. Other investigators $[14,7]$ have found that $c<0$ if adhesion in friction predominates and that $c>0$ if the adhesion component is small and friction is caused by deformation. The values of $c$ for the dental restorative materials were $0.54,0.19,0.23,0.17,0.31$ and 0.25 for materials $\mathrm{A}-\mathrm{F}$, respectively. From Table 2 it can be seen that $c=n-1$; therefore, $\mu=K N^{n-1}$.

\section{Conclusions}

When a diamond hemispherical slider was slid across the surface of filled and unfilled dental restorative resins and dental amalgams in the presence of 
distilled water, the frictional force $F$ was related to the normal load $N$ by the equation $F=K N^{n}$. The constant $K$ was a function of the modulus of elasticity $E$, yield strength $\sigma_{\mathrm{y}}$, fracture toughness $R$ and slider diameter $D$ as given by $K=A R^{2} / \sigma_{\mathrm{y}} E^{n} D^{2 n}$. The sliding friction under these test conditions was caused by deformation with adhesion playing a minor role. The coefficient of friction $\mu$ for the dental restorative materials followed an equation of the form $\mu=K N^{n-1}$ in which $n-1>0$.

\section{Acknowledgment}

This investigation was supported by USPHS Research Grant DE-03416 from the National Institute of Dental Research, National Institutes of Health, Bethesda, Md. 20014.

\section{References}

1 M. W. Pascoe and D. Tabor, The friction and deformation of polymers, Proc. R. Soc. London, Ser. A, 235 (1956) 185 - 191.

2 D. Tabor and D. E. Wayne Williams, The effect of orientation on the friction of poly tetrafluoroethylene, Wear, 4 (1961) $391-400$.

3 J. F. Archard, Elastic deformation and the laws of friction, Proc. R. Soc. London, Ser. A, 243 (1957) $190-205$.

4 D. Tabor, Friction, lubrication and wear of synthetic fibers, Wear, $1(1957 / 58) 5-23$.

5 E. Rabinowicz (ed.), Friction and Wear of Materials, Wiley, New York, 1965, pp. $10-31$.

6 E. Hornbogen, The role of fracture toughness in the wear of metals, Wear, 33 (1975) $251-259$.

7 D. Atack and D. Tabor, The friction of wood, Proc. R. Soc. London, Ser. A, 246 (1958) $539-555$.

8 J. M. Powers, J. C. Roberts and R. G. Craig, Surface failure of commercial and experimental restorative resins, J. Dent. Res., 55 (1976) $432-436$.

9 J. C. Roberts, J. M. Powers and R. G. Craig, Wear of dental amalgam, J. Biomed. Mater. Res., 11 (1977) 513 - 523.

10 J. M. Powers and R. G. Craig, Wear of fluorapatite single crystals: I. A method for quantitative evaluation of wear, J. Dent. Res., 51 (1972) $168-176$.

11 J. M. Powers and R. G. Craig, Wear of fluorapatite single crystals: II. Frictional behavior, J. Dent. Res., 51 (1972) $605-610$.

12 J. C. Roberts, J. M. Powers and R. G. Craig, The fracture toughness of composite and unfilled restorative resins, J. Dent. Res., in the press.

13 J. C. Roberts, J. M. Powers and R. G. Craig, The fracture toughness and critical strain energy release rate of dental amalgam, J. Mater. Sci., 1977, in the press.

14 W. J. Duncan (ed.), Physical Similarity and Dimensional Analysis, Edward Arnold, London, 1953, 87 - 97.

15 A Manual of Elementrary Statistics using MIDAS, Statistical Research Laboratoty, University of Michigan, Ann Arbor, Michigan, 1975, p. 301.

16 D. E. Grenoble and L. J. Katz, The pressure dependence of the elastic constants of dental amalgam, J. Biomed. Mater. Res., 5 (1971) 489 - 502.

17 D. R. Hall, W. T. Nakayma, D. E. Grenoble and J. L. Katz, Elastic constants of three representative dental cements, J. Dent. Res., 52 (1973) 390. 